\title{
Pseudobloqueo de conducción en vasculitis aislada del sistema nervioso periférico
}

\section{Pseudo-conduction block in nonsystemic vasculitic neuropath}

\author{
R.M. Pabón' ${ }^{1}$, L. Gila ${ }^{1}$, J. Urriza ${ }^{1}$, L. Imirizaldu ${ }^{1}$, M. Arrechea ${ }^{2}$, F. Lacruz ${ }^{3}$
}

\section{RESUMEN}

Fundamento. La vasculitis aislada del sistema nervioso periférico (VASNP) afecta selectivamente a los vasa nervorum, expresándose generalmente como una mononeuropatía múltiple. Presentamos un caso de VASNP confirmado histológicamente, destacando los hallazgos neurofisiológicos en fase aguda.

Observación clínica. Mujer de 36 años con parestesias y debilidad en mano derecha seguidas de paresia para la dorsiflexión del pie izquierdo. El primer estudio neurofisiológico mostraba amplitud reducida del potencial motor del mediano derecho con estímulos proximales. Un segundo estudio mostraba signos de lesión axonal en varios nervios, incluyendo el mediano derecho.

Conclusiones. La lesión isquémica aguda de un nervio puede dar lugar a un patrón electroneurográfico de bloqueo de conducción, como en el mediano derecho del caso descrito. Este fenómeno es conocido como "pseudobloqueo", dado su carácter transitorio, con evolución a un patrón de neuropatía axonal. La sospecha de VASNP requiere estudios neurofisiológicos seriados para una correcta tipificación de los patrones lesionales.

Palabras clave. Vasculitis. Mononeuropatía múltiple. Electromiografía. Biopsia de nervio sural. Degeneración walleriana.

\begin{abstract}
Summary. Introduction. Nonsystemic vasculitic neuropathy (NSVN) is an inflammatory disorder of the vasa nervorum which usually is expressed as a mononeuritis multiplex. We present a patient with NSVN with histological confirmination focused on the neurophysiological findings at the early stages.
\end{abstract}

Case report. A 36 years-old woman presented with paresthesia and weakness in her right hand followed by left footdrop. The first neurophysiologic examination showed low amplitude of the right median nerve (RMN) CMAP with proximal stimulation. A second examination showed signs of axonal damage in several nerves, including the RMN.

Conclusions. The acute ischemic damage of a nerve can give a pattern of conduction block in the electroneurographic study as in the RMN of the presented case. This phenomenon is referred as "pseudo-conduction block", since it is transient and evolves towards a definite pattern of axonal neuropathy. When a vasculitic neuropathy is suspected, repeated neurophysiologic studies are necessary in order to ensure a proper (appropriate) characterization of the lesional patterns.

Key words. Vasculitis. Mononeuritis multiplex. Electromyography. Sural nerve biopsy. Wallerian degeneration.
1. Servicio de Neurofisiología Clínica. Hospital Virgen del Camino. Pamplona.

2. Servicio de Anatomía Patológica. Hospital de Navarra. Pamplona

3. Servicio de Neurología. Hospital de Navarra.

Este caso fue presentado como póster en la "XLIV Reunión Nacional de la Sociedad Española de Neurofisiología" (Toledo, 18-21 de octubre de 2006).

Recepción: 30 de abril de 2009

Aceptación provisional: 7 de mayo de 2009

Aceptación definitiva: 11 de junio de 2009

\section{Correspondencia}

Rocío Milena Pabón Meneses

Servicio de Neurofisiología Clínica

Hospital Virgen del Camino

Ronda Cendea de Zizur, $58-2^{\circ} \mathrm{C}$

31010 Barañain. Navarra

Tfno.+34679802102

E-mail: drarociomilena@hotmail.com 


\section{INTRODUCCIÓN}

La vasculitis aislada del sistema nervioso periférico (VASNP) es un proceso inflamatorio que afecta selectivamente a los vasa nervorum, cuya forma clínica más característica es la mononeuropatía múltiple de curso agudo o subagudo ${ }^{1,2}$. Aunque es una enfermedad rara, la VASNP y la neuropatía asociada a poliarteritis nodosa y poliangiítis microscópica son las formas de neuropatía vasculítica más frecuentemente en la literatura $^{3}$, representando cada una alrededor del $25 \%$ en grandes series ${ }^{4}$. Se estima que la sensibilidad de la biopsia de nervio sural es del $50-60 \%$, ${ }^{2,3}$ los estudios neurofisiológicos muestran signos de afectación de troncos nerviosos periféricos con distribuciones topográficas variables. Las alteraciones neurofisiológicas típicas traducen la degeneración axonal aguda de los infartos nerviosos. Menos frecuente es el patrón de bloqueo de conducción (BC) en fase aguda ${ }^{1,2}$, que en la mayoría evolucionan a un patrón de degeneración axonal, denominados "pseudobloqueos de conducción" ${ }^{5,6}$. Presentamos un caso de VASNP confirmado histopatológicamente en el que se objetivaron datos neurofisiológicos de pseudobloqueo de conducción.

\section{CASO CLÍNICO}

Mujer de 36 años, sin antecedentes, que inicia con parestesias en dedo pulgar de la mano derecha seguidas, 2 días después, de debilidad para el movimiento de pinza. Dos días más tarde, aparece paresia para la dorsiflexión del pie izquierdo y parestesias dolorosas en el dorso del mismo. Fue ingresada al cabo de una semana del inicio la semana de evolución, observandose paresia grado 3 en la escala del Medical Research Council (MRC) del oponente y del abductor corto del pulgar (ACP) derechos y grado 4 MRC de los músculos dependientes del ciático poplíteo externo (CPE) izquierdo junto con hipoestesia táctil y dolorosa en primer y segundo dedos de la mano derecha y en dorso del pie izquierdo. Mostraba un buen estado general sin fiebre, lesiones cutáneas ni adenopatías. Cinco días después de su ingreso aparecen nuevas alteraciones neurológicas consistentes en paresia grado 3 MRC de deltoides y de tríceps braquial izquierdo y parestesias en cara externa del brazo izquierdo y ambas pantorrillas.

Los estudios complementarios realizados incluyeron velocidad de sedimentación globular (VSG), hemograma, bioquímica elemental de sangre y orina, estudio de liquido cefalorraquídeo (LCR), estudio de coagulación que incluyó plaquetas, volumen plaquetar medio, tiempo de protrombina, Ratio Internacional Normalizada (INR) y fibrinógeno, proteinograma, inmunoglobulinas, fracciones C3 y C4 del complemento, crioglobulinas, anticuerpos antinucleares (ANA), anticuerpos anti citoplasma del neutrófilo (ANCA), anticuerpos anticardiolipina IgG e IgM y antigangliósidos GM1 y GD1b, factor reumatoide, serología a lúes (RPR), Borrelia burgdorferi y hepatitis B y C, Mantoux, electrocardiograma (ECG), radiografía de tórax, resonancia magnetica (RM) cerebral, torácica y abdominal. Los hallazgos de estas exploraciones resultaron dentro del rango de la normalidad.

Durante el ingreso se realizan dos estudios neurofisiológicos que se detallan en la tabla 1 . El primero realizado a los 9 días del inicio de los síntomas, destacó la ausencia de potencial motor (PM) del CPE izquierdo y una reducción de la amplitud del PM del mediano derecho con estímulos en muñeca, codo y axila respecto a la del obtenido con estimulación palmar (Fig. 1a). El electromiograma (EMG) del ACP derecho mostraba un patrón de máximo esfuerzo simple con escasos potenciales de unidad motora (PUMs) de características normales y sin actividad espontánea. En el tibial anterior (TA) izquierdo no se registro actividad espontánea ni voluntaria. En el segundo estudio (Tabla 1) realizado a los 15 días de evolución, se objetivaron cambios en la conducción sensitiva de varios nervios, que previamente no habían mostrado alteraciones. Éstos incluyeron, reducción de la amplitud del potencial sensitivo del nervio peroneal superficial izquierdo, ausencia de respuestas sensitivas en los nervios surales y en el mediano derecho al estímular en tercer dedo y disminución de la amplitud de los obtenidos desde primer y segundo dedos. Como en el primer estudio, no se obtuvo PM del CPE izquierdo. La amplitud de los PMs del mediano derecho estaba reducida, incluyendo la correspondiente a la estimulación en la palma (Fig. 1b), que había disminuido respecto a la del estudio previo. En el EMG se registró actividad espontánea en forma de fibrilaciones y ondas positivas, abundantes en el ACP derecho y escasas en TA y pedio izquierdos. Como en el estudio anterior, el reflejo $\mathrm{H}$ y las ondas $\mathrm{F}$ de cubitales, tibiales posteriores, mediano izquierdo y CPE derecho fueron normales y no se observaron bloqueos proximales de la conducción motora en el mediano izquierdo y en ambos cubitales, obteniéndose valores normales hasta punto de Erb.

Se realizó una biopsia del nervio sural izquierdo, que mostró degeneración axonal e infiltrado inflamatorio perivascular compatible con infarto por vasculitis (Fig. 2). 


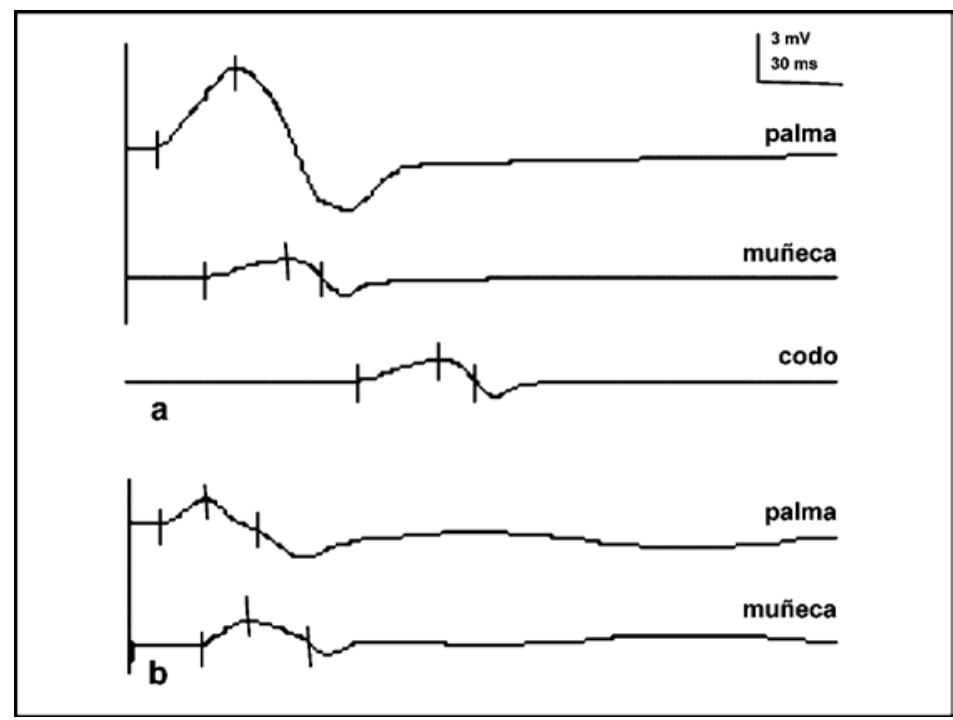

Figura 1. Electroneurografía motora del nervio mediano derecho con registro de superficie sobre eminencia tenar. A la derecha de las señales se indican los puntos de estimulación. a) Estudio efectuado a los 9 días de evolución. Con estimulación en muñeca y codo los potenciales motores muestran una amplitud notoriamente inferior respecto a la del obtenido con estimulación en la palma, sin aumento de la dispersión temporal. b) El estudio realizado 6 días después muestra una reducción de la amplitud del potencial obtenido con estimulación en la palma, similar al de la estimulación en muñeca.

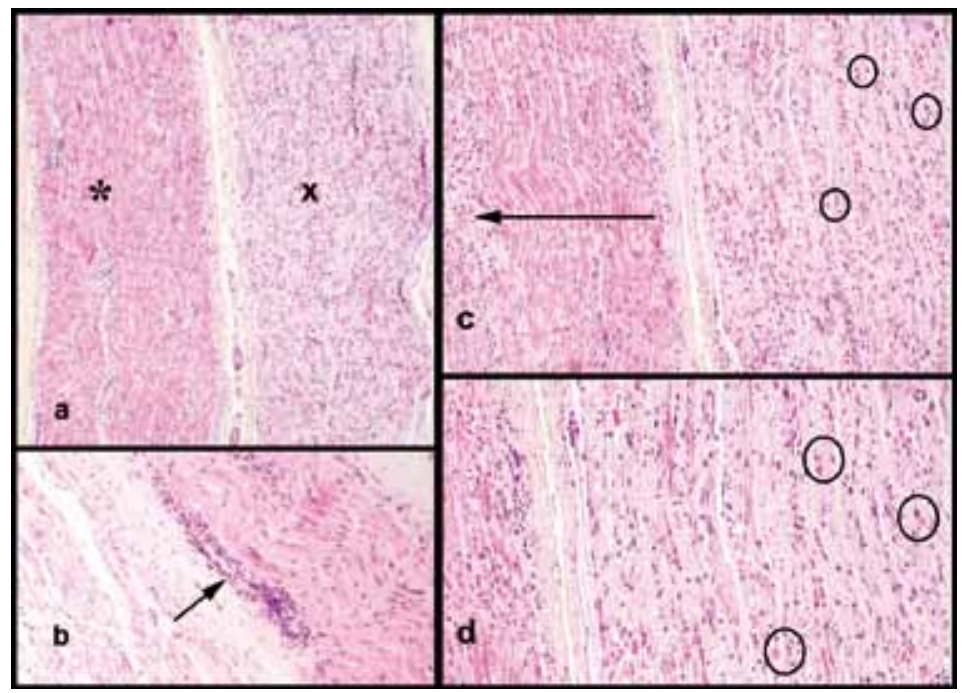

Figura 2. Imágenes histológicas del nervio sural mediante tinción con hematoxilina-eosina. a) Fascículo nervioso intacto (asterisco) y fascículo infartado (aspa) vistos a bajo aumento (x 200). b) Infiltrado linfocitario en la pared de un vaso epineural (flecha) (x 400). c) A la izquierda un fascículo relativamente conservado, con infiltrados inflamatorios en el perineuro (flecha); a la derecha un fascículo infartado, visto a mayor aumento, que contiene esferoides axonales y ovoides de mielina (círculos) correspondientes a degeneración walleriana de las fibras nerviosas (x 400). d) Detalle de los cambios por degeneración walleriana señalados en c). 
Tabla 1. Resultados de los estudios neurofisiológicos. Exploraciones realizadas a los 9 días, 15 días y 9 meses de evolución. Valores patológicos en negrita.

\begin{tabular}{|c|c|c|c|c|c|c|c|}
\hline \multicolumn{8}{|c|}{ Conducción motora } \\
\hline \multirow{2}{*}{ Nervio } & \multirow{2}{*}{ Estímulo } & \multicolumn{2}{|c|}{$1^{\text {a }}$ Exploración } & \multicolumn{2}{|c|}{$2^{\text {a }}$ Exploración } & \multicolumn{2}{|c|}{$3^{\mathrm{a}}$ Exploración } \\
\hline & & VCM & Amp. & VCM & Amp. & VCM & Amp. \\
\hline \multirow[t]{4}{*}{ Mediano D } & Palma & & 5,0 & & 1,1 & & \\
\hline & Muñeca & 38 & 1,3 & 36 & 1,1 & & 0,7 \\
\hline & Codo & 44 & 1,4 & 39 & 1,0 & 35 & 0,7 \\
\hline & Axila & 47 & 1,3 & & & & \\
\hline \multirow[t]{4}{*}{ Mediano I } & Palma & & 15,0 & & & & \\
\hline & Muñeca & 53 & 10,0 & & 9,0 & & 10,0 \\
\hline & Codo & 57 & 10,0 & 53 & 8,6 & 50 & 9,0 \\
\hline & Erb & 66 & 9,0 & 72 & 8,4 & & \\
\hline \multirow[t]{3}{*}{ Cubital D } & Muñeca & & 7,0 & & 6,5 & & 13,0 \\
\hline & Codo & 62 & 6,0 & 55 & 6,0 & 52 & 13,0 \\
\hline & Erb & 61 & 5,5 & 67 & 5,5 & & \\
\hline \multirow[t]{3}{*}{ Cubital I } & Muñeca & & 6,0 & & 7,0 & & 12,0 \\
\hline & Codo & 66 & 6,0 & 57 & 6,6 & 53 & 11,0 \\
\hline & Erb & 69 & 6,0 & 71 & 6,0 & & \\
\hline \multirow[t]{2}{*}{ CPE D } & Tobillo & & 4,0 & & 3,0 & & 3,0 \\
\hline & Rodilla & 46 & 4,0 & 42 & 3,0 & 45 & 3,0 \\
\hline \multirow[t]{2}{*}{ CPE I } & Tobillo & & - & & - & & - \\
\hline & Rodilla & & - & & - & & - \\
\hline $\mathrm{CPE}^{*} \mathrm{D}$ & Rodilla & & 6,6 & & & & \\
\hline $\mathrm{CPE}^{*} \mathrm{I}$ & Rodilla & & 1,7 & & 1,2 & & 2,0 \\
\hline \multirow[t]{2}{*}{ TP D } & Tobillo & & 6,0 & & 5,5 & & 6,0 \\
\hline & Rodilla & 46 & 5,0 & 41 & 5,0 & 42 & 4,0 \\
\hline \multirow[t]{2}{*}{ TP I } & Tobillo & & 6,0 & & 5,0 & & 7,0 \\
\hline & Rodilla & 46 & 5,5 & 43 & 4,5 & 43 & 5,0 \\
\hline \multirow[t]{2}{*}{ Radial $^{* *} \mathrm{D}$} & Codo & & & & 3,5 & & \\
\hline & Brazo & & & 52 & 3,2 & & \\
\hline \multirow[t]{2}{*}{ Radial $^{* *}$ I } & Codo & & & & 3,0 & & \\
\hline & Brazo & & & 53 & 2,5 & & \\
\hline Circunflejo D & Erb & & & & 3,2 & & \\
\hline Circunflejo I & Erb & & & & 3,0 & & \\
\hline
\end{tabular}




\begin{tabular}{|c|c|c|c|c|c|c|c|}
\hline \multicolumn{8}{|c|}{ Conducción sensitiva } \\
\hline \multirow{2}{*}{ Nervio } & \multirow{2}{*}{ Estímulo } & \multicolumn{2}{|c|}{ 1aㅡ Exploración } & \multicolumn{2}{|c|}{$2^{\mathrm{a}}$ Exploración } & \multicolumn{2}{|c|}{$3^{\text {a Exploración }}$} \\
\hline & & VCS & Amp. & VCS & Amp. & VCS & Amp. \\
\hline \multirow[t]{3}{*}{ Mediano D } & $1^{\text {er }}$ dedo & & & 52 & 3,0 & & \\
\hline & $2^{\circ}$ dedo & & & 52 & 3,0 & 50 & 3,0 \\
\hline & $3^{\text {er }}$ dedo & 56 & 14,0 & & - & & - \\
\hline \multirow[t]{3}{*}{ Mediano I } & $1^{\text {er }}$ dedo & & & 50 & 16,0 & & \\
\hline & $2^{\circ}$ dedo & 59 & 13,0 & 57 & 9,0 & 54 & 8,0 \\
\hline & $3^{\text {er }}$ dedo & & & 57 & 10,0 & & \\
\hline Cubital D & $5^{\circ}$ dedo & 57 & 9,0 & 55 & 5,0 & 51 & 6,0 \\
\hline Cubital I & $5^{\circ}$ dedo & 62 & 12,0 & 57 & 8,0 & 50 & 8,0 \\
\hline Radial sup. D & & & & 51 & 24 & & \\
\hline Radial sup. I & & & & 50 & 21 & & \\
\hline Sural D & Pantorrilla & 48 & 8,0 & & - & 48 & 3,0 \\
\hline Sural I & Pantorrilla & 49 & 9,0 & & - & & (biopsiado) \\
\hline Peroneo sup. D & Pierna & 53 & 10,0 & 42 & 8,0 & 44 & 9,0 \\
\hline Peroneo sup. I & Pierna & 46 & 9,0 & 43 & 3,0 & 43 & 1,0 \\
\hline
\end{tabular}

\begin{tabular}{|l|c|c|c|c|c|c|c|c|c|}
\hline \multicolumn{10}{|c|}{ Electromiografía } \\
\hline \multirow{2}{*}{ Músculo } & \multicolumn{3}{|c|}{$\mathbf{1}^{\mathbf{a}}$ Exploración } & \multicolumn{3}{|c|}{$\mathbf{2}^{\mathbf{a}}$ Exploración } & \multicolumn{3}{|c|}{$\mathbf{3}^{\mathbf{a}}$ Exploración } \\
\cline { 2 - 11 } & $\mathbf{A E}$ & $\mathbf{P U M s}$ & $\mathbf{P M E}$ & $\mathbf{A E}$ & $\mathbf{P U M s}$ & $\mathbf{P M E}$ & $\mathbf{A E}$ & $\mathbf{P U M s}$ & PME \\
\hline ACP D & 0 & $\mathrm{~N}$ & $\downarrow \downarrow \downarrow$ & +++ & $\mathrm{N}$ & $\downarrow \downarrow \downarrow$ & ++ & PF & $\downarrow \downarrow$ \\
\hline PID D & 0 & $\mathrm{~N}$ & $\mathrm{~N}$ & & & & & & \\
\hline TA I & 0 & $\mathbf{0}$ & $\mathbf{0}$ & + & $\mathbf{0}$ & $\mathbf{0}$ & ++ & PF & $\downarrow \downarrow \downarrow$ \\
\hline TA D & 0 & $\mathrm{~N}$ & $\mathrm{~N}$ & & & & 0 & $\mathrm{~N}$ & $\mathrm{~N}$ \\
\hline PLL I & 0 & $\mathrm{~N}$ & $\downarrow \downarrow \downarrow$ & & & & & & \\
\hline Pedio I & & & & + & $\mathbf{0}$ & $\mathbf{0}$ & + & $\mathbf{0}$ & $\mathbf{0}$ \\
\hline Tríceps I & & & & 0 & $\mathrm{~N}$ & $\downarrow$ & + & PF & $\downarrow$ \\
\hline ECD I & & & & 0 & $\mathrm{~N}$ & $\mathrm{~N}$ & & & \\
\hline Deltoides I & & & & 0 & $\mathrm{~N}$ & $\mathrm{~N}$ & & & \\
\hline
\end{tabular}

D: derecho; I: izquierdo; CPE: ciático poplíteo externo; TP: tibial posterior; *: registro de superficie sobre tibial anterior; **: registro de superficie sobre extensor propio del índice; sup.: superficial; VCM: velocidad de conducción motora en m/s; VCS: velocidad de conducción sensitiva en $\mathrm{m} / \mathrm{s}$; Amp.: amplitud, de los potenciales motores en mV y sensitivos en $\mu \mathrm{V}$; - no se obtiene; ACP: abductor corto del pulgar; PID: primer interóseo dorsal; TA: tibial anterior; PLL: peroneo lateral largo; ECD: extensor común de los dedos; AE: actividad espontánea; PUMs: potenciales de unidad motora; PEM: patrón de máximo esfuerzo; N: normal; 0: no se registra; +: escasa; ++: moderada; +++: abundante; PF: polifásicos; $\downarrow$ : ligeramente deficitario; $\downarrow \downarrow$ : moderadamente deficitario; $\downarrow \downarrow \downarrow$ : marcadamente deficitario (patrón simple).

Se instauró tratamiento con $60 \mathrm{mg} /$ día de prednisona, con mejoría subjetiva de la debilidad muscular y de las parestesias dolorosas. Posteriormente se redujo gradualmente la dosis de prednisona. A los 9 meses, $45 \mathrm{mg}$ a días alternos, mejoría de parestesias en las extremidades inferiores y de fuerza proximal en la extremidad superior izquierda. Persistía déficit sensitivomotor en la mano derecha y paresia marcada en territorio del CPE izquierdo. Un tercer estudio neurofisiológico realizado a los 9 meses (Tabla 1) no mostro cambios significativos respecto al anterior, excepto la reaparición del potencial sensitivo de baja amplitud en el nervio sural de- 
recho. En el EMG persistía moderada actividad espontánea en ACP derecho y TA izquierdo, con patrones de máximo esfuerzo marcadamente deficitarios y PUMs con signos de reinervación activa, irregulares (polifásicos y politurns) e inestables.

\section{DISCUSIÓN}

Los hallazgos clínicos y neurofisiológicos de esta paciente cumplen los criterios diagnósticos de VASNP, propuestos por Dyck y col $^{1}$ que incluyen afectación exclusiva del sistema nervioso periférico (SNP) sin evidencia clínica o analítica de afectación sistémica y presencia de degeneración axonal y cambios microvasculíticos de las arteriolas epineurales en la biopsia de nervio periférico.

La afectación del SNP en la VASNP, como en otras vasculitis, consiste en una neuropatía axonal multifocal de instauración agua o subaguda por infartos isquémicos de los troncos nerviosos. Ante una afectación aguda del SNP, debe considerarse un conjunto de entidades de etiología diversa cuyos tratamientos son asimismo muy diferentes. Para la orientación del diagnóstico, es necesaria una correcta caracterización de las alteraciones del SNP a través del estudio neurofisiológico.

Los patrones de alteraciones neurofisiológicas traducen los mecanismos fisiopatológicos subyacentes de los distintos síndromes. Tales patrones aparecen bien definidos en lesiones plenamente instauradas, pero en estadios iniciales, los cambios parciales pueden inducir interpretaciones erróneas. Para evitarlo se debe valorar conjuntamente la clínica y los hallazgos neurofisiológicos, en estudios seriados.

El caso descrito presenta un patrón electroneurográfico de $\mathrm{BC}$ en el primer estudio que pudo atribuirse a una patología de tipo desmielinizante, cuando en realidad se trataba de una alteración transitoria de origen isquémico.

La afectación vascular de un nervio periférico produce un daño axonal focal expresado electroneurográficamente por una reducción de las amplitudes de los potenciales (motores o sensitivos) con velocidades de conducción normales o escasamente disminuidas. Si el infarto abarca la totalidad de las fibras de un nervio no se obtiene potencial evocado motor o sensitivo. En el EMG se observa inicialmente un patrón de máximo esfuerzo deficitario o nulo y a los 10-14 días de la lesión aparece actividad espontánea de denervación aguda en forma de fibrilaciones y ondas positivas ${ }^{7}$. En nuestro caso estos hallazgos típicos de neuropatía axonal aguda se encontraron en los nervios mediano derecho, CPE y peroneal superficial izquierdos en el segundo estudio neurofisiologico realizado a los 15 días de evolución. En el primer estudio realizado a los 9 días, los resultados no eran concluyentes de una afectación axonal, observándose en el nervio mediano derecho una marcada caída de la amplitud del PM al estimular en puntos proximales (muñeca, codo y axila) respecto al PM obtenido con estimulación distal (palmar). Esta disminución de la amplitud en puntos proximales, superior al $50 \%$ y sin signos de dispersión temporal ni variaciones anatómicas que lo justifiquen, configura un patrón electroneurográfico de $\mathrm{BC}^{8-10}$.

La fisiopatología del BC consiste en un fallo de la conducción del potencial de acción en un punto de una fibra nerviosa, manteniéndose normal la conducción en el segmento distal a dicho punto ${ }^{8}$. Los BC típicamente indican desmielinización focal con indemnidad estructural del axón ${ }^{11} \mathrm{y}$ son frecuentes en diferentes neuropatías desmielinizantes como la polirradiculoneuropatía desmielinizante inflamatoria aguda (AIDP o síndrome de Guillain-Barré clásico $)^{12,13}$, una variante de neuropatía motora aguda con bloqueos de conducción ${ }^{14}$, la polineuropatía inflamatoria desmielinizante crónica (CIDP) $)^{10}$, la neuropatía inflamatoria desmielinizante multifocal (síndrome de Lewis-Sumner $)^{15}$ y la neuropatía hereditaria con labilidad a las parálisis por presión ${ }^{4}$. También se observan BCs por alteración de los mecanismos iónicos de despolarización de las fibras nerviosas de origen disinmune, como en la neuropatía motora multifocal ${ }^{16}$, o tóxico, como se ha descrito en la polineuropatía por n-hexano ${ }^{17}$. 
En la afectación vascular de los nervios periféricos, como en la VASNP, además de patrón típico de neuropatía axonal, se han observado $\mathrm{BC}$ en estudios experimentales y clínicos. En modelos animales de isquemia de troncos nerviosos pueden apreciarse alteraciones según la intensidad y la duración de la isquemia y el tiempo transcurrido tras la misma, que abarcan desde trastornos metabólicos y cambios estructurales inicialmente reversibles (edema axonal, desmielinización) en la isquemia leve y transitoria, hasta degeneración axonal walleriana tras infarto completo establecido $^{18}$. La degeneración walleriana por isquemia condiciona una pérdida axonal predominante en el centro de los fascículos nerviosos, pero en las zonas circundantes pueden verse fibras que conservan los axones intactos y muestran desmielinización segmentaria y paranodal ${ }^{18-21}$. En concordancia con estos hechos, en modelos experimentales de neuropatía isquémicas sin infarto completo se han observado BCs reversibles $^{22}$ y bloqueos persistentes ${ }^{19}$, igualmente descritos en el terreno clínico ${ }^{16,23-26}$.

Los BC descritos son de 3 tipos según su curso evolutivo: reversibles, persistentes y transitorios (Tabla 2). Los $\mathrm{BC}$ reversibles se atribuyen a alteraciones metabólicas o estructurales leves que se recuperan en breve plazo ${ }^{22,23,25}$, estos se han observado en vasculitis necrotizantes. Los $\mathrm{BC}$ persistentes son más raros y se deben a cambios desmielinizantes, como algunos casos descritos en pacientes con angiopatía necrotizante $^{23,24,27} \mathrm{o}$ anemia falciforme ${ }^{28}$. Aunque es un hallazgo atípico, en series de VASNP la presencia de $\mathrm{BC}$ es de un 10 a un $25 \%$ de los $\operatorname{casos}^{1,2}$, siendo el más frecuente en las neuropatías isquémicas los BC transito$\operatorname{rios}^{5-7,16}$. Los $\mathrm{BC}$ transitorios o pseudobloqueos son aquellos $\mathrm{BC}$ presentes durante un lazo corto de tiempo en estudios realizados precozmente, tras el cual aparece un patrón de lesión axonal aguda. Trabajos experimentales de nervios periféricos han mostrado la secuencia de estos cambios, observándose a los 3-8 días tras la lesión isquémica un patrón de $\mathrm{BC}$ que cambia a los 9-14 días a un patrón axonal por infarto completo. Esto explica que en nuestro caso descrito encontráramos un patrón de $\mathrm{BC}$ en el nervio mediano los 9 días siguientes a la lesión y a los 15 días, el paso a un patrón de lesión axonal. Este patrón de BC transitorio en fases precoces de lesiones axonales agudas ha sido referido como "pseudobloqueo de conducción" por algunos autores $^{5,6}$ y por otros como "bloqueo con solución de continuidad del axón" ("axon non-continuity conduction block") ${ }^{29,30}$.

Tabla 2. Tipos de bloqueo de conducción nerviosa en vasculitis y otras neuropatías isquémicas.

\begin{tabular}{lllll}
\hline \multicolumn{1}{c}{ Tipo de bloqueo } & \multicolumn{1}{c}{ Fisiopatología } & \multicolumn{1}{c}{ Evolución } & \multicolumn{1}{c}{$\begin{array}{c}\text { Cambios } \\
\text { neurofisiológicos }\end{array}$} & Frecuencia \\
\hline $\begin{array}{l}\text { Transitorios o } \\
\text { pseudobloqueos }\end{array}$ & $\begin{array}{l}\text { Infarto nervioso en } \\
\text { fase aguda }\end{array}$ & $\begin{array}{l}\text { A degeneración } \\
\text { walleriana en 7-9 } \\
\text { días }\end{array}$ & $\begin{array}{l}\text { Sustitución por } \\
\text { patrón de lesión } \\
\text { axonal }\end{array}$ & 10-25\% \\
& $\begin{array}{l}\text { Trastornos } \\
\text { metabólicos de las } \\
\text { fibras nerviosas } \\
\text { condicionados por } \\
\text { isquemia leve y } \\
\text { transitoria }\end{array}$ & $\begin{array}{l}\text { Restablecimiento } \\
\text { de la conducción } \\
\text { normal en días o } \\
\text { semanas }\end{array}$ & $\begin{array}{l}\text { Normalización de } \\
\text { los parámetros de } \\
\text { conducción }\end{array}$ & Ocasionales \\
& $\begin{array}{l}\text { Afectación } \\
\text { estructural de las } \\
\text { vainas de mielina }\end{array}$ & $\begin{array}{l}\text { Desmielinización } \\
\text { segmentaria } \\
\text { definitiva o con } \\
\text { recuperación en } \\
\text { meses-años }\end{array}$ & $\begin{array}{l}\text { Patrón de bloqueo } \\
\text { permanente; } \\
\text { eventual } \\
\text { nomalización tardía }\end{array}$ & Excepcionales \\
\hline
\end{tabular}


La secuencia y plazos temporales de los cambios fisiopatológicos descritos en las neuropatías isquémicas deben tenerse en cuenta para una adecuada valoración de los hallazgos neurofisiológicos en el contexto de una neuropatía aguda. Así pues, ante la sospecha de neuropatía aguda por vasculitis y presencia de BCs es necesario realizar estudios neurofisiológicos seriados para conseguir una correcta tipificación de los patrones lesionales que asegure el diagnóstico sindrómico.

\section{BIBLIOGRAFÍA}

1. Dyck PJ, Benstead TJ, Conn DL, Stevens JC, WinDEBANK AJ, Low PA. Nonsystemic vasculitic neuropathy. Brain 1987; 110: 843-853.

2. Collins MP, Periquet Mi, Mendell JR, Sahenk Z, NAGARAJA HN, KISSEL JT. Nonsystemic vasculitic neuropathy: Insights from a clinical cohort. Neurology 2003; 61: 623-630.

3. CASAS JM, ACHA MV. Etiopatogenia de la arteritis de células gigantes. Anal Sist Sanit Navar 2003; 26: 43-61.

4. Collins MP, Periquet, Mi. Non-systemic vasculitic neuropathy. Curr Opin Neurol 2004; 17 : 587-598.

5. McCluskey L, Feinberg D, Cantor C, Bird S. Pseudo-conduction block in vasculitic neuropathy. Muscle Nerve 1999; 22: 1361-1366.

6. Sandbrink F, Klion AD, Floeter MK. Pseudoconduction block in a patient with vasculitic neuropathy. Electromyogr Clin Neurophysiol 2001; 41: 195-202.

7. Chaudhry V, Cornblath DR. Wallerian degeneration in human nerves: serial electrophysiological studies. Muscle Nerve 1992; 15: 687693.

8. AAEE glossary of terms in clinical electromyography. Muscle Nerve 1987; 10: G1-60.

9. Cornblath DR, Sumner AJ, Daube J, Guilliat RW, BROWN WF, PARRY GJ et al. Conduction block in clinical practice. Muscle Nerve 1991; 14: 869-871.

10. Ad Hoc Subcommittee of the American Academy of Neurology AIDS Task Force. Research criteria for the diagnosis of chronic inflammatory polyneuropathy (CIDP). Neurology 1991; 41: 617-618.

11. Feasby TE, Brown WF, Gilbert JL, Hahn AF. The pathological basis of conduction block in hu- man peripheral neuropathies. J Neurol Neurosurg Psychiatry 1985; 48: 239-244.

12. Mills KR, Murray NMF. Proximal conduction block in early Guillain-Barré syndrome. Lancet $1985 ; 2: 659$.

13. Berger AR, Logigian EL, Shahani BT. Reversible proximal conduction block underlies rapid recovery in Guillain-Barré syndrome. Muscle Nerve 1988; 11: 1039-1042.

14. Capasso M, Caporale CM, Pomilio F, Gandolfi P, Lugaresi A, Uncini A. Acute motor conduction block neuropathy. Another Guillain-Barré syndrome variant. Neurology 2003; 61: 617-622.

15. Lewis RA, Sumner AJ, Brown MJ, Asbury AK. Multifocal demyelinating neuropathy with persistent conduction block. Neurology 1982; 32: 958-966.

16. Traba A, Esteban A. Neuropatía motora con bloqueos de conducción. Neurología 1993; 8: 59-65.

17. Chang AP, England JD, García CA, Sumner AJ. Focal conduction block in n-hexane polyneuropathy. Muscle Nerve 1998; 21: 964-969.

18. NukAda H, Dyck PJ. Acute ischemia causes axonal stasis, swelling, attenuation, and secondary demyelination. Ann Neurol 1987; 22: 311-318.

19. Fowler CJ, Guilliatt RW. Conduction velocity and conduction block after experimental ischaemic nerve injury. J Neurol Sci 1981; 52 : 221-238.

20. NuKADA H, Dyck PJ. Microsphere embolization of nerve capillaries and fiber degeneration. Am J Pathol 1984; 115: 275-287.

21. Korthals JK, Gieron MA, MaKi T, Belsole RJ. Peripheral nerve demyelination after transient ischemia. Neurology 1984; 34 Suppl 1: 168.

22. PARry GJ, LinN DJ. Transient focal conduction block following experimental occlusion of the vasa nervorum. Muscle Nerve 1986; 9 : 345-348.

23. Ropert A, Metral S. Conduction block in neuropathies with necrotizing vasculitis. Muscle Nerve 1990; 13: 102-105.

24. JamiesonPW, Guiliani MJ, Martínez AJ. Necrotizing angiopathy presenting with multifocal conduction block. Neurology 1991; 41: 442444.

25. Magistris MR, Kohler A, Estade M. Conduction block in vasculitic neuropathy. Eur Neurol 1994; 34: 283-285.

26. Chico A, Llobet J, Corcoy R, Cuatrecases M, Dourado M, Illa I. Polineuropatía sensitiva y bloqueo de conducción como primera ma- 
nifestación de una poliarteritis nodosa. Rev Neurol 1995; 23: 679-681.

27. BecKett VL, Dinn JJ. Segmental demyelination in rheumatoid arthritis. Q J Med 1972; 41: 71-80.

28. Shields RW, Harris JW, Clark M. Mononeuropathy in sickle cell anemia: anatomical and pathophysiological basis for its rarity. Muscle Nerve 1991; 14: 370-374.
29. Wilbourn AJ, Levin KH. Ischemic neuropathy. En Brown WF, Bolton CF, eds. Clinical electromyography ( $2^{\mathrm{a}}$ ed). Stoneham: Butterworth-Heinemann; 1993. p. 369-390.

30. Briemberg HR, Levin K, Amato AA. Multifocal conduction block in peripheral nerve vasculitis. J Clin Neuromusc Dis 2002; 3: 153158. 
